\title{
Deep Dehydration of Antarctic Lichen Leptogium puberulum Hue Observed by NMR and Sorption Isotherm
}

\author{
H. HarańCzyK ${ }^{a, *}$, M. Bacior ${ }^{a}$, P. JastrzȩBska ${ }^{a}$ And M.A. Olech ${ }^{b, c}$ \\ ${ }^{a}$ Institute of Physics, Jagiellonian University \\ Reymonta 4, 30-059 Kraków, Poland \\ ${ }^{b}$ Institute of Botany, Jagiellonian University \\ Kopernika 27, 31-501 Kraków, Poland \\ ${ }^{c}$ Department of Antarctic Biology, Polish Academy of Sciences \\ Ustrzycka 10, 02-141 Warszawa, Poland
}

\begin{abstract}
Hydration kinetics, sorption isotherm, and proton free induction decays are measured for Leptogium puberulum Hue thalli hydrated from gaseous phase. Very tightly, tightly and loosely bound water fraction are distinguished. The hydration dependence of mobile NMR signal component is non-linear and fitted well by rational function, which suggest the presence of water soluble solid (presumably carbohydrate) fraction in thallus structures of $L$. puberulum.
\end{abstract}

PACS numbers: $82.56 . \mathrm{Na}$

\section{Introduction}

Some Antarctic lichen species may survive extremely harsh environmental conditions, desiccation stress and frost [1-3]. They perform active photosynthesis below $0^{\circ} \mathrm{C}[4,5]$, below ice nucleation of their cellular fluids [6], and can hydrate from gaseous phase to the hydration level sufficient to initiate active photosynthetic activity, e.g. Usnea aurantiaco-atra (Jacq.) Bory and Ramalina terebrata Hook. f. \& Taylor start photosynthesis at the hydration level equal to $\Delta m / m_{0} \geq 0.2-0.3$ [7], whereas the hydration level from gaseous phase equals for foliose Umbilicaria aprina Nyl. $\Delta m / m_{0}=0.85$ (at $\left.p / p_{0}=100 \%\right)[8]$, and for fruticose Usnea antarctica Du Rietz to $\Delta m / m_{0}=1.2[9,10]$. The dehydration of intracellular space below ice crystal formation threshold is one of the ways of dealing with freezing by extremophilic organism. Thus, it is very interesting to observe water uptake by lichen from gaseous phase.

The unusual effectiveness of lichen hydration from gaseous phase turn our attention to the process of initial stages of thallus hydration, various bound water fractions, the number and distribution of water binding sites, sequence and kinetics of their saturation and their mobility. We used hydration kinetics, sorption isotherm and high power proton nuclear magnetic relaxometry for the investigations of fruticose lichen Leptogium puberulum thalli.

* corresponding author; e-mail: hubert.haranczyk@uj.edu.pl

\section{Materials and methods}

Leptogium puberulum Hue thalli were collected during Antarctic summer in maritime Antarctic, Antarctic Peninsula, King George Island, from the site localized close to Polish Antarctic H. Arctowski Station [11]. The site position was $62^{0} 10^{\prime} \mathrm{S}$ and $58^{0} 25^{\prime} \mathrm{W}$. Air-dry thalli were stored at room temperature with the hydration level $\Delta m / m_{0}=0.072 \pm 0.011$, where $m_{0}$ is the dry mass of the sample, and $\Delta m$ is mass of water taken up (determined gravimetrically).

Before the hydration experiments thalli were incubated for $200 \mathrm{~h}$ over silica gel (at relative humidity, $p / p_{0}=$ $0 \%$ ), dehydrating to the hydration level $\Delta m / m_{0}=$ $0.012 \pm 0.003$. Dehydration kinetics was single exponential, with the dehydration time $t_{\mathrm{d}}=(12.1 \pm 2.6) \mathrm{h}$.

The hydration courses were performed from the gaseous phase with the controlled humidity, at room temperature $\left(t=22^{\circ} \mathrm{C}\right)$, over the surface of $\mathrm{H}_{3} \mathrm{PO}_{4}\left(p / p_{0}=\right.$ $9 \%$ ), over the surfaces of saturated solutions of $\mathrm{KC}_{2} \mathrm{H}_{3} \mathrm{O}_{2}$ $\left(p / p_{0}=23 \%\right), \mathrm{CaCl}_{2}\left(p / p_{0}=32 \%\right), \mathrm{K}_{2} \mathrm{CO}_{3}(44 \%)$, $\mathrm{Na}_{2} \mathrm{Cr}_{2} \mathrm{O}_{7}\left(p / p_{0}=52 \%\right), \mathrm{NH}_{4} \mathrm{NO}_{3}(63 \%), \mathrm{Na}_{2} \mathrm{~S}_{2} \mathrm{O}_{3}$ $(76 \%), \mathrm{K}_{2} \mathrm{CrO}_{3}(88 \%), \mathrm{Na}_{2} \mathrm{SO}_{4}(93 \%), \mathrm{K}_{2} \mathrm{SO}_{4}(97 \%)$, and over a water surface $\left(p / p_{0}=100 \%\right)$.

After completing the hydration courses, the dry mass of the thallus was determined after heating at $70^{\circ} \mathrm{C}$. Higher temperatures were not used as they may cause the decomposition of some organic constituents of thallus [12]. Suggested by Gaff, 1977, duration of heating equal to $72 \mathrm{~h}$ was not sufficient in case of Leptogium puberulum thallus, as for longer heating times measurable decrease in mass was recorded. To perform proper de- 
hydration of the $L$. puberulum thallus to dry mass the minimal heating time $175 \mathrm{~h}$ was needed, as the dehydration time constant equals $(35 \pm 15) \mathrm{h}$.

Hydration kinetics experiments were performed on one sample per given relative humidity. The air-dry thalli used for the NMR measurements were chopped and placed in NMR tubes, and then the hydration procedure was performed. The NMR measurements were performed on five samples. Every sample covered a part of the hydration range which overlapped with other parts.

Proton free induction decays were recorded on WNS HB-65 high power relaxometer (Waterloo NMR Spectrometers, St. Agatha, Ontario, Canada). The resonance frequency was $30 \mathrm{MHz}$ (at $B_{0}=0.7 \mathrm{~T}$ ); the transmitter power was $400 \mathrm{~W}$; the pulse length $\pi / 2=$ $1.4 \mu \mathrm{s}$. The high power of the pulse allowed us to observe the total proton signal. Free induction decays (FIDs) were acquired using Compuscope 2000 card in an IBM clone computer, controling the spectrometer, and averaged over 2000 accumulations. Repetition time was $2.003 \mathrm{~s}$. The data obtained were analyzed using the one-dimensional, FID analyzing procedure of the two-dimensional (in time domain) NMR signal-analyzing program CracSpin written at the Jagiellonian University, Cracow [13].

\section{Results}

The hydration courses for L. puberulum thalli performed from the gaseous phase at the relative humidity, $p / p_{0}$, controlled between $9 \%$ and $76 \%$, were fitted well by a single exponential function (see Fig. 1)

$$
\Delta m / m_{0}=A_{0}^{\mathrm{h}}+A_{1}^{\mathrm{h}}\left[1-\exp \left(-t / t_{1}^{\mathrm{h}}\right)\right],
$$

where $\Delta m / m_{0}$ is the relative mass increase, $A_{0}^{\mathrm{h}}$ is the very tightly bound water fraction level saturating for the hydration times shorter than the first measuring point (10 $\mathrm{min}), A_{1}^{\mathrm{h}}$ is the saturation level for the fast component (solely observed in this relative humidity range), at incubation $\left(p / p_{0}=0 \%\right)$, and $t_{1}^{\mathrm{h}}$ is the corresponding hydration time constant.

At the relative humidity higher than $76 \%$ the slow hydration component appeared and the hydration courses were fitted well by the two-exponential function (Fig. 1)

$$
\begin{aligned}
& \Delta m / m_{0}=A_{0}^{\mathrm{h}}+A_{1}^{\mathrm{h}}\left[1-\exp \left(-t / t_{1}^{\mathrm{h}}\right)\right] \\
& \quad+A_{2}^{\mathrm{h}}\left[1-\exp \left(-t / t_{2}^{\mathrm{h}}\right)\right],
\end{aligned}
$$

where $A_{0}^{\mathrm{h}}$ is the amplitude of the very fast component, $A_{1}^{\mathrm{h}}$ and $A_{2}^{\mathrm{h}}$ are the saturation hydration levels for fast and slow component, $t_{1}^{\mathrm{h}}$ and $t_{2}^{\mathrm{h}}$ are the corresponding hydration time constants.

The samples hydrated at $p / p_{0}=100 \%, 97 \%$, and $93 \%$ were attacked by fungi during hydration courses. The analysis showed the presence of Cosmopolitan molds, characteristic for Central Europe.

Very tightly bound water component amplitude equals $A_{0}^{\mathrm{h}}=0.008 \pm 0.004$, which was significantly smaller than the one observed for majority of other lichen species

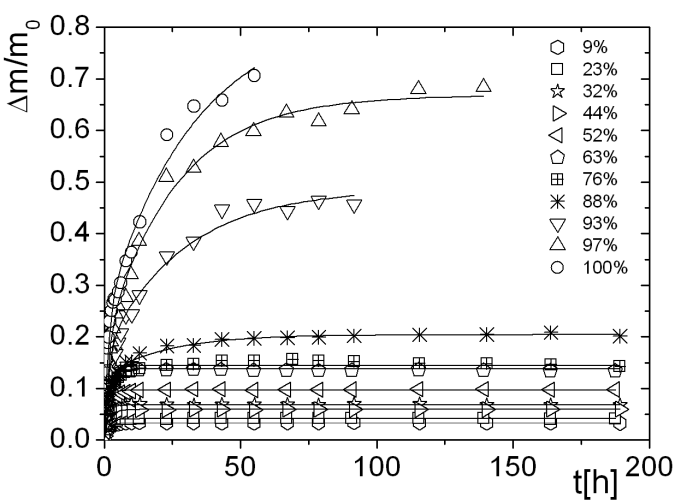

Fig. 1. Hydration kinetics of L. puberulum performed from the gaseous phase (the target humidities are marked in the inset). The errors are within the plot symbols.

$[8,10,14]$. This bound water component is differentiated by short time of saturation (and, thus, binding strength). It is removed by dehydration for $72 \mathrm{~h}$ at $+70^{\circ} \mathrm{C}$.

Tightly bound water hydration time constant was equal to $t_{1}^{\mathrm{h}}=(1.6 \pm 0.3) \mathrm{h}$, whereas the amplitude did not saturate with the hydration level, as it is observed in other lichen thalli, e.g. lichens from genera Usnea, Cladonia, or some species from Umbilicaria. It will be discussed later.

Well defined loosely bound component appeared at $p / p_{0}=88 \%$, similarly to the one observed in foliose $U m$ bilicaria aprina [8]. The hydration time constant was equal for slow component, $t_{2}^{\mathrm{h}}=(25.8 \pm 3.2) \mathrm{h}$, whereas the amplitude progressively increased with the increasing humidity.

Two exponential functions of hydration kinetics are characteristic of lichen thalli exposed to fast changes in weather conditions in their habitats (fruticose lichens [10], or foliose Umbilicaria aprina collected from glacial stream in Schirmacher Oasis on Antarctic continent [8]), whereas for foliose thalli, better protected from rapid temperature changes, e.g. foliose Umbilicaria antarctica, or fruticose Caloplaca regalis (Vain.) Zahlbr., single exponential kinetics is observed [14].

The total saturation hydration level,

$$
\begin{aligned}
& C^{\mathrm{h}}=A_{0}^{\mathrm{h}}+A_{1}^{\mathrm{h}}+A_{2}^{\mathrm{h}} \\
& \text { or } \quad C^{\mathrm{h}}=A_{0}^{\mathrm{h}}+A_{1}^{\mathrm{h}},
\end{aligned}
$$

in the absence of slow component, was taken for construction of sorption isotherm.

For Leptogium puberulum thalli the sorption isotherm reveals a sigmoidal form (Fig. 2), which is well fitted by the Dent [15] and/or BET [16] model. Both these models distinguish two types of water binding sites on the surfaces of the thallus, namely, (i) "primary" binding sites (directly to the thallus surfaces); and (ii) "secondary", usually weaker (to the previously bound water molecules, or to the thallus water binding sites with much smaller bond strengths). The difference is that the BET model 
takes a fixed value of the ratio of the number of binding sites covered by $n$ water molecules to that covered by $n-1$ water molecules, $b=S_{n} /\left.S_{n-1}\right|_{h=1}=1$ (which is an artificial assumption), whereas in the Dent model this ratio may be varied between 0 and 1 (to some extent simulating clustering effect)

$$
C^{\mathrm{h}}(h)=\frac{\Delta M}{m_{0}} \frac{b_{1} h}{(1-b h)\left(1+b_{1} h-b h\right)},
$$

where $h$ is relative humidity, $p / p_{0}$, expressed in absolute units, $\Delta M / m_{0}$, is the mass of the water fraction saturating primary binding sites, and the reciprocal of $b_{1}$ parameter is $S_{0} /\left.S_{1}\right|_{h=1}=1 / b_{1}$, which is a contribution of not populated primary water binding sites in units of sites populated by one water molecule at $h=1$.

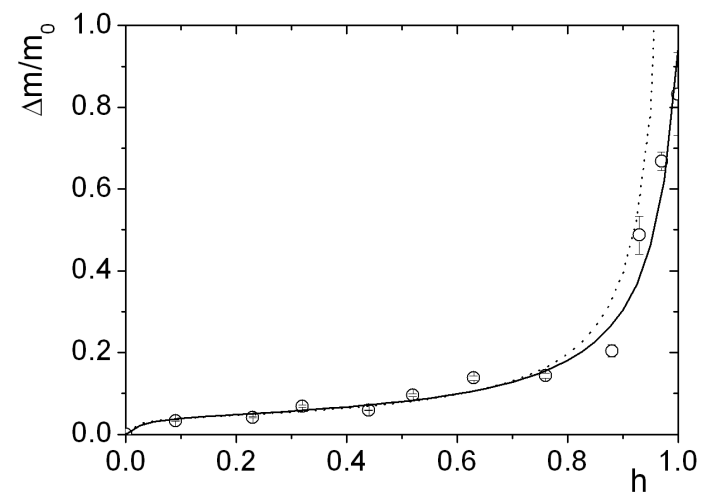

Fig. 2. The sorption isotherm for L. puberulum (solid line - the Dent model, dashed line - BET model). The values of $h\left(=p / p_{0}\right)$ represent the relative humidity and the values of relative mass increase, $\Delta m / m_{0}$, are taken as the saturation values $C^{\mathrm{h}}$ from Eq. (2a), or Eq. (2b).

To test the applicability of the sorption model the sorption isotherm is usually presented in parabolic form (Fig. 3). The parabolic form for the Dent isotherm is expressed as

$$
\frac{h}{\Delta m / m_{0}}=A+B h-C h^{2},
$$

where parameters $\frac{\Delta M}{m_{0}}, b, b_{1}$ are connected with $A, B, C$ by the formulae

$$
\begin{aligned}
& b=\frac{\sqrt{B^{2}+4 A C}-B}{2 A}, \quad b_{1}=\frac{B}{A}+2 b, \\
& \frac{\Delta M}{m_{0}}=\frac{1}{A b_{1}} .
\end{aligned}
$$

The parabolic form of the BET isotherm is described by

$$
\frac{h}{\Delta m / m_{0}}=A+B h-(A+B) h^{2} .
$$

For $h=1$ BET parabola equals 0, whereas the Dent model yields non-zero positive value. Thus, the difference from zero at $h=1$ is the measure of the applicability of the Dent model. For Leptogium puberulum thalli the sorption isotherm is much better described by the Dent model (see Fig. 3). Other test of applicability of the Dent model, the value of $b$ parameter equals
$0.955 \pm 0.008$, which is an elevated value compared to other fruticose thalli [14]. The mass of water saturating primary binding sites was $\Delta M / m_{0}=0.043 \pm 0.007$, which is a little lower value than for other fruticose lichens from the genera Usnea [14, 10], or Cladonia [14]. The contribution from empty binding sites at $h=1$ is given by $1 / b_{1}=(2.59 \pm 2.25) \%$ (expressed as percentage), which is comparable to that for other fruticose lichens, eg. for Cladonia mitis Sandst. $1 / b_{1}=2.44 \%$, or for Caloplaca regalis $1 / b_{1}=1.93 \%[14]$.

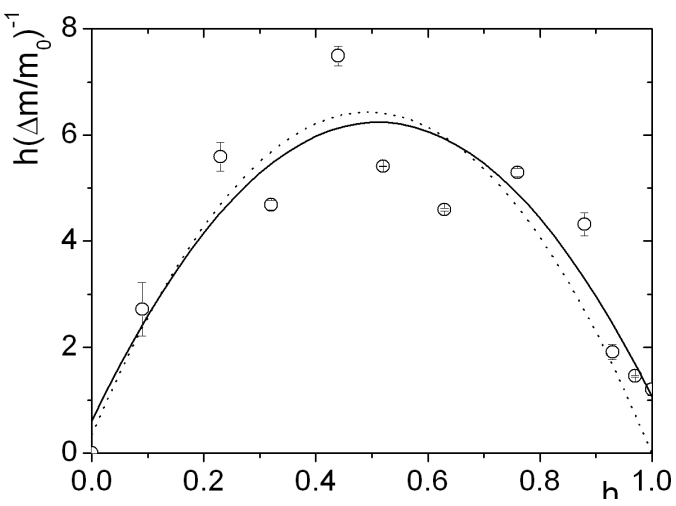

Fig. 3. Parabolic form of Dent and BET model (open circles $=$ experimental data, solid line $=$ fitted Dent model, dotted line $=$ BET model).

Free induction decays for protons of Leptogium puberulum thallus, at lower hydration levels, are well fitted by the superposition of one Gaussian component, with the amplitude $S$, coming from the solid matrix of thallus and one exponential component with amplitude equal to $L_{1}$ (see Fig. 4)

$$
\begin{aligned}
& \mathrm{FID}(t)=S \exp \left[-\left(\frac{t}{T_{2 S}^{*}}\right)^{2}\right] \\
& +L_{1} \exp \left(-\frac{t}{T_{2 L_{1}}^{*}}\right) .
\end{aligned}
$$

For higher hydration levels $\left(\Delta m / m_{0}>0.232\right)$ two exponential components, $L_{1}$ and $L_{2}$, coming from water tightly and loosely bound on the surfaces of thallus, respectively, are observed

$$
\begin{aligned}
& \mathrm{FID}(t)=S \exp \left[-\left(\frac{t}{T_{2 S}^{*}}\right)^{2}\right]+L_{1} \exp \left(-\frac{t}{T_{2 L_{1}}^{*}}\right) \\
& \quad+L_{2} \exp \left(-\frac{t}{T_{2 L_{1}}^{*}}\right),
\end{aligned}
$$

where $T_{2 S}^{*}$ is the proton spin-spin relaxation time of solid component taken as the 1/e-value of Gaussian solid signal, and $T_{2 L_{1}}^{*}$ and $T_{2 L_{2}}^{*}$ are the relaxation times of proton mobile (liquid) fractions $L_{1}$ and $L_{2}$, respectively.

The solid signal for Leptogium puberulum was nearly Gaussian in form (the contribution of sinc function was hardly seen in residual function (see Fig. 4b)), with the $T_{2 S}^{*}$ value (Fig. 5), which did not change much with the hydration level, suggested that the structure and dynam- 


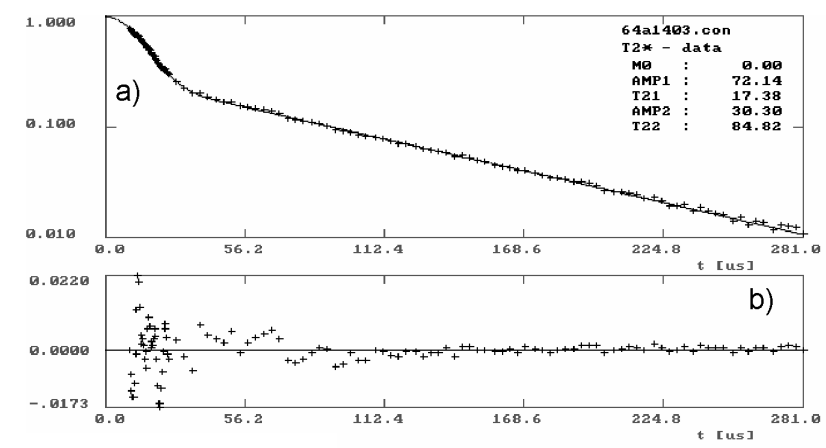

Fig. 4. (a) Proton free induction decay recorded for L. puberulum thalli at $30 \mathrm{MHz}$; the pulse length $\pi / 2=$ $1.4 \mu \mathrm{s}$. The relative mass increase was $\Delta m / m_{0}=0.210$. $Y$-axis shows the normalized signal amplitude. The solid line represents a least squares fit of Eq. (7a) to the data. (b) The residual function calculated as the difference between the fitted and recorded values of the FID signal, which form any recorded point does not exceed $2.2 \%$.

ics of thallus solid matrix is hardly modified by hydration process. Thus, we used the amplidude, $S$, of Gaussian solid component as an unit for scaling the signals from liquid components.

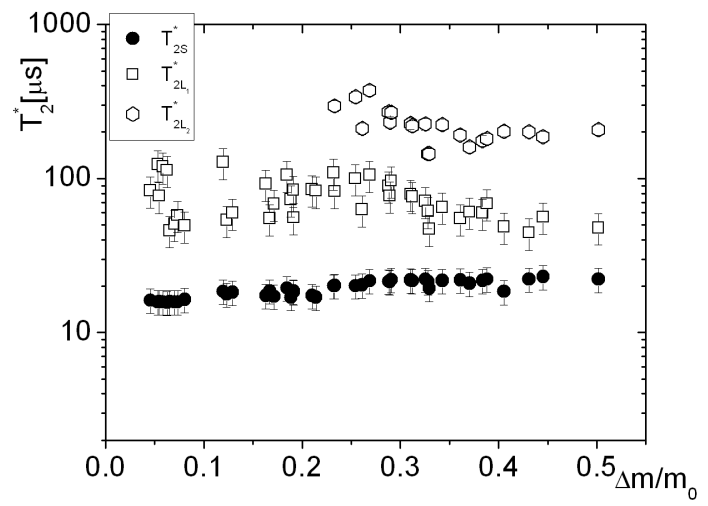

Fig. 5. The hydration dependence of proton FID relaxation times for L. puberulum (solid Gaussian, S, component - closed circles, tightly bound water, $L_{1}$, component - open squares, and loosely bound water, $L_{2}$, component - open hexagons).

The observed water fractions are differentiated by their mobility and, thus, by their binding and/or proximity to the thallus surfaces. This means that either intracellular or extracellular water fraction usually contribute to both these water fractions. The $T_{2 L_{1}}^{*} \approx 100 \mu$ s of the $L_{1}$ signal component is characteristic of tightly bound water fraction in lichen thalli $[1,10,17]$, and for many other biological systems [18-20]. The $L_{2}$ signal with longer $T_{2 L_{2}}^{*}$, but shortened by $B_{0}$ inhomogeneities, comes from water loosely bound on thallus surface, and for higher hydration levels, from free water fraction (Fig. 5).
The hydration dependence of total liquid signal, expressed in the units of solid, $\left(L_{1}+L_{2}\right) / S$, is shown in Fig. 6 and is well fitted by the rational function

$$
\begin{aligned}
& \frac{L_{1}+L_{2}}{S}\left(\Delta m / m_{0}\right) \\
& =\frac{(-0.41 \pm 0.10)+(4.77 \pm 0.43) \Delta m / m_{0}}{1+(-1.51 \pm 0.04) \Delta m / m_{0}} .
\end{aligned}
$$

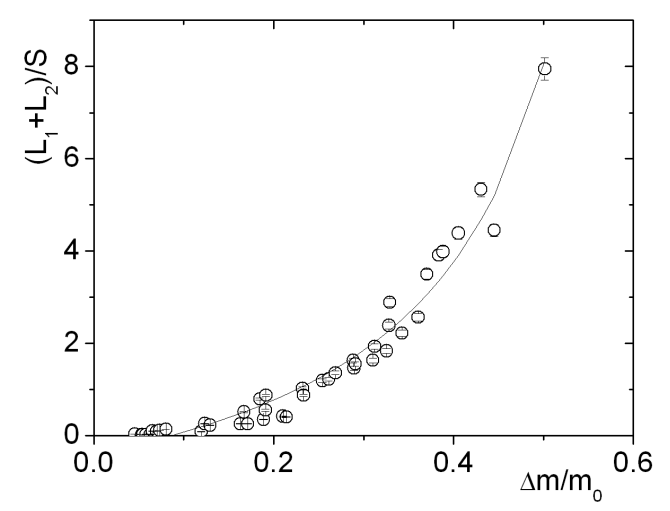

Fig. 6. The total mobile proton signal, $\left(L_{1}+L_{2}\right) / S$, hydration dependence for $L$. puberulum. The solid line was calculated from Eq. (8).

If water soluble solid fraction is present in the sample, the hydration dependence of the liquid-to-solid signal amplitude ratio is described by the rational function [18], so far as whole fraction of water soluble solid fraction is not dissolved. For higher hydration levels, the liquid-to-solid hydration dependence becomes linear [20], which was not observed for initial hydration steps for the thallus of Leptogium puberulum.

The amplitude of loosely bound signal, $L_{2} / S$, increases linearly with increasing mass, $\Delta m / m_{0}$, of water added, and is fitted well by the linear function (Fig. 7)

$$
L_{2} / S=(14.47 \pm 1.15) \Delta m / m_{0}-(3.67 \pm 0.40) .
$$

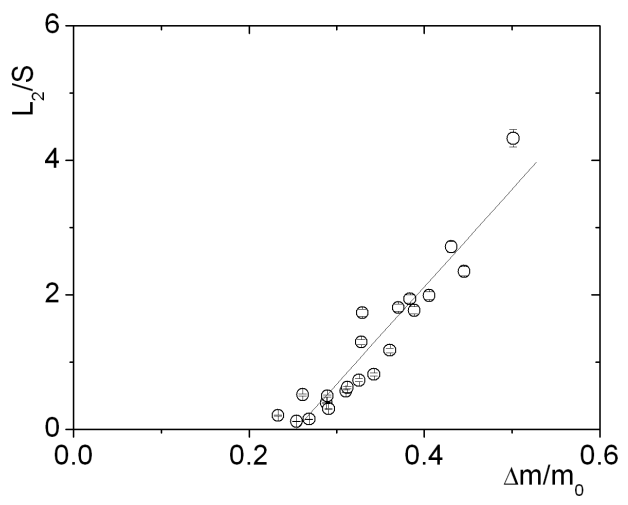

Fig. 7. The $L_{2} / S$ hydration dependence for $L$. puberulum. The solid line was calculated from Eq. (9).

That the dependence of $L_{2} / S$ is well simplified by linear function does not mean the linear dependence of $L_{2}$ 
fraction. However, as $L_{2} / S$ hydration dependence may be simplified by linear function means that the loosely bound water fraction is less influenced by water soluble solid fraction.

\section{Discussion}

The non-linear form of $L / S$ hydration dependence was observed in horse chestnut bast and was fitted well by rational function within the hydration range where water soluble solid fraction (sucrose) was still in solid phase; for higher hydration levels the linear hydration dependence was observed [20]. Lichen thallus may hydrate to relatively high level (eg. Usnea sphacelata to $\Delta m / m_{0}=1.6$ [21], Lasalia pustulata to 4.0, and Umbilicaria spadochroa to 5.0 [22], and the change of rational-to- linear function describing the hydration dependence for lichen may occur at physiologic hydration levels, however, in this paper we focused on hydration level close to the restart of photosynthesis process [23-26]. For hydration from gaseous phase of $L$. puberulum thalli the overall hydration level is not sufficient to dissolve the whole amount of water soluble solid (presumably carbohydrate) fraction, thus, the change in form of $L / S$ hydration dependence was not observed. The accumulation of monosaccharides (as glucose, fructose, mannitol, and arabitol) in lichen mycobionts may be the adaptative feature for growing in dry conditions [27], however, the relatively high carbohydrate level is detected also in lichens populating wet habitats (e.g. $32 \mathrm{mg} / \mathrm{g}$ dry wt. in dry habitat, and 34 $\mathrm{mg} / \mathrm{g}$ dry wt. in wet habitat, Windmill Islands region of continental Antarctica [28]), and the proportion of carbohydrate fractions is not much changed [29]. Characteristic of glass state formation at extremely low hydration levels of sugar samples is a "beat" pattern in FID function [30]. However, for L. puberulum the "beat" pattern was much smaller than in other lichen samples [8], where differential scanning calorimetry (DSC) experiments do not reveal glassy transition. This suggests that if glassy transition in L. puberulum cells takes place, it refers to small volumes of thallus. Loosely bound water fraction, $L_{2}$, was not affected by water soluble solid fraction, which suggests that major part of $L_{2}$ signal may come from extracellular spaces of thallus.

\section{References}

[1] H. Harańczyk, S. Gaździński, M. Olech, in: New Aspects in Cryptogamic Research, Contribution in Honour of Ludger Kappen. Bibl. Lichenol. 75, 265 (2000).

[2] H. Harańczyk, J. Grandjean, M. Olech, Colloids \& Surfaces, B: Biointerfaces 28, 239 (2003).
[3] H. Harańczyk, J. Grandjean, M. Olech, M. Michalik, Colloids \& Surfaces, B: Biointerfaces 28, 251 (2003).

[4] L. Kappen, M. Breuer, M. Bölter, Polar Biology 11 , 393 (1991).

[5] L. Kappen, B. Schroeter, C. Scheidegger, M. Sommerkorn, G. Hestmark, Adv. Space Res. 18, 119 (1996).

[6] M.R. Worland, W. Block, H. Oldale, Cryo-Lett. 17, 31 (1996).

[7] L. Kappen, in: Lichen Physiology and Cell Biology, Ed. D.H. Brown, Plenum Press, New York 1985, p. 41.

[8] H. Harańczyk, M. Bacior, M.A. Olech, Antarctic Sci. 20, 527 (2008).

[9] L. Kappen, M. Breuer, Antarctic Sci. 3, 273 (1991).

[10] H. Harańczyk, A. Pietrzyk, A. Leja, M.A. Olech, Acta Phys. Pol. A 109, 411 (2006).

[11] B. Schroeter, M. Olech, L. Kappen, W. Heitland, Antarctic Sci. 7, 251 (1995).

[12] D.F. Gaff, Oecologia (Berl.) 31, 95 (1977).

[13] W. Wȩglarz, H. Harańczyk, J. Phys. D, Appl. Phys. 33, 1909 (2000).

[14] H. Harańczyk, On Water in Extremely Dry Biological Systems, Wydawnictwo Uniwersytetu Jagiellońskiego, Kraków 2003.

[15] R.W. Dent, Textile Res. J. 47, 145 (1977).

[16] S. Brunauer, P.H. Emmett, E. Teller, J. Am. Chem. Soc. 60, 309 (1938).

[17] H. Harańczyk, S. Gaździński, M.A. Olech, New Phytologist 138, 191 (1998).

[18] H. Harańczyk, A. Leja, K. Strzałka, Acta Phys. Pol. A 109, 389 (2006).

[19] H. Harańczyk, K. Strzałka, G. Jasiński, K. Mosna-Bojarska, Coll. Surf. A 115, 47 (1996).

[20] H. Harańczyk, W.P. Wȩglarz, S. Sojka, Holzforschung 53, 299 (1999).

[21] L. Kappen, Polar Biol. 1, 249 (1983).

[22] L. Kappen, B. Schroeter, G. Hestmark, J.B. Winkler, Bot. Acta 109, 292 (1996).

[23] A. Bertsch, Planta (Berl.) 70, 46 (1966).

[24] A. Bertsch, Planta (Berl.) 68, 157 (1966).

[25] O.L. Lange, Flora, Abt. B 158, 324 (1969).

[26] T.H. Nash III, A. Reiner, B. Demmig-Adams, E. Kilian, W.M. Kaiser, O.L. Lange, New Phytol. 116, 269 (1990).

[27] N. Hamada, K. Okazaki, M. Shinozaki, Bryologist 97, 176 (1994).

[28] D.R. Melick, R.D. Seppelt, Crypt. Bot. 4, 212 (1994).

[29] D.R. Melick, R.D. Seppelt, Bryologist 97, 13 (1994).

[30] L.M. Crowe, Comp. Bioch. Physiol. A 131, 505 $(2002)$.

str. 519, szpalta lewa, linia 3 od gry, ma byf: as an unit . 